\title{
Chronic diarrhea, bile acids, and Clostridia
}

\author{
Julian R.F. Walters ${ }^{1,2}$ and Julian R. Marchesi ${ }^{1,3}$ \\ 'Division of Digestive Diseases, Department of Metabolism, Digestion, and Reproduction, Faculty of Medicine, Imperial College London, London, United Kingdom. ${ }^{2}$ Gastroenterology, Division of Medicine \\ and Integrated Care, Imperial College Healthcare NHS Trust, London, United Kingdom. ${ }^{3}$ School of Biosciences, Cardiff University, Cardiff, United Kingdom.
}

\begin{abstract}
Excessive fecal bile acid (BA) loss causes symptoms in a large proportion of people diagnosed with irritable bowel syndrome with diarrhea, a common functional bowel disorder. This BA diarrhea (BAD) results from increased hepatic synthesis of BAs, with impaired negative feedback regulation by the ileal hormone fibroblast growth factor 19 (FCF19). In this issue of the $\mathrm{JCl}$, Zhao et al. investigated BA metabolism, including fecal BAs, serum BAs, and FGF19, in patients and controls. They identified associations between fecal bacterial BA metabolism and specific microbiota, especially Clostridium scindens. These findings have been tested in a mouse model using microbiota transplants and antibiotic treatment. This group of organisms has potential as a biomarker for BAD and to be a target for therapy.
\end{abstract}

\section{Pathophysiology of the} functional bowel disorders

Bile acids (BAs) are increasingly recognized as major factors in the pathophysiology of the functional bowel disorders with diarrhea $(1,2)$. Recent guidelines recommend investigation of patients with chronic diarrhea to detect the presence of $\mathrm{BA}$ diarrhea (BAD) $(3,4)$. Such patients may meet the diagnostic criteria for irritable bowel syndrome with predominant diarrhea (IBS-D) or functional diarrhea (FD), depending on how they report abdominal pain (1), but they all experience frequent, soft or liquid stools, with urgency and often fecal incontinence, with a response to BA sequestrants (5). Diagnostic delay is common, as the recognition of BAD by physicians is poor, and there is limited availability of diagnostic tests, such as the nuclear medicine ${ }^{75} \mathrm{Se}$-homocholic acid taurine (SeHCAT) test $(4,6)$. SeHCAT is unavailable in the US, which has led to the development of alternative blood or fecal tests (7). The frequency of BAD is estimated at $28 \%$ in IBS-D or FD, giving a population prevalence of $1 \%(8-10)$.
The precise causes of this idiopathic, primary BAD (PBAD) have largely been unclear. BAs are mostly reabsorbed in the ileum and undergo enterohepatic circulation. BA malabsorption, which is clearly present in ileal Crohn's disease or resection, is usually not found in PBAD. An alternative cause for the excess fecal BA loss is increased hepatic BA synthesis $(6,11)$. BAs are synthesized from cholesterol by the enzyme cholesterol $7 \alpha$-hydroxylase (CYP7A1). Raised levels of the BA intermediate $7 \alpha-\mathrm{OH}-4$-cholesten-3one (C4) demonstrate the increased activity of the classical synthesis pathway, where CYP7A1 is rate limiting. This enzyme is regulated via negative feedback by the ileal hormone fibroblast growth factor 19 (FGF19). Serum FGF19 levels are usually low in PBAD, and are inversely correlated with C4 (12). In ileal tissue, BA and other agonists bind to the farnesoid $\mathrm{X}$ receptor (FXR) to stimulate FGF19 transcription (13). In PBAD, there is evidence for impaired FGF19 production from the ileum (14). Further, PBAD is associated with certain polymorphisms in genes (FGFR4 and KLB) that affect the hepatic response to FGF19 (15).

\section{Related Article: p. 438}

Conflict of interest: The authors have declared that no conflict of interest exists.

\section{A possible cause of PBAD}

In this issue of the JCI, Zhao et al. investigated the relevance of the BA/FXR signaling pathway and the effects of microbial metabolism of BAs in relation to the possible cause of PBAD (16). Starting with an unselected population of 345 Chinese adults who met Rome IV diagnostic criteria for IBS-D (1), the authors identified approximately $25 \%$ of these with excess total fecal BAs (called the BA ${ }^{+}$IBS-D group by Zhao et al.). This figure is a similar proportion to the IBS-D patients reported elsewhere with PBAD (8-10). Importantly, the authors showed that the BA-positive group, when compared with the BA-negative group, also showed raised serum $\mathrm{C} 4$ and total BA levels, with lower FGF19. Total fecal BAs were associated with increased symptom severity, increased BA synthesis, and impaired FGF19 feedback, making the Zhao et al. study patients representative of those studied previously (16).

BA profile differences in serum and fecal samples of the BA-positive group suggest that there are differences in the BA-transforming microbiota in PBAD. In the serum, BAs conjugated with glycine or taurine and the free unconjugated liver-derived (primary) BAs, cholic acid (CA) and chenodeoxycholic acid (CDCA), predominated. CDCA and glycochenodeoxycholic acid (GCDCA) were increased, and the secondary BA, ursodeoxycholic acid (UDCA) and its conjugated form, glycoursodeoxycholic acid (GUDCA), were also particularly higher in the BApositive group. Similarly, in feces, increases in the total of most BAs, and the proportions of CA, CDCA, UDCA, and 7-ketodeoxycholic acid (7-KDCA) were found. Relevantly, identifying raised levels of unconjugated primary BAs (CA and CDCA) in fecal samples has been proposed as a novel diagnostic method to identify PBAD (7).

Intestinal bacteria possess specialized BA-modifying enzymes, and consequently are integral to BA metabolism. Deconjugation, i.e., removing glycine or taurine, by choloylglycine hydrolase (also known as bile salt hydrolase) encoded 


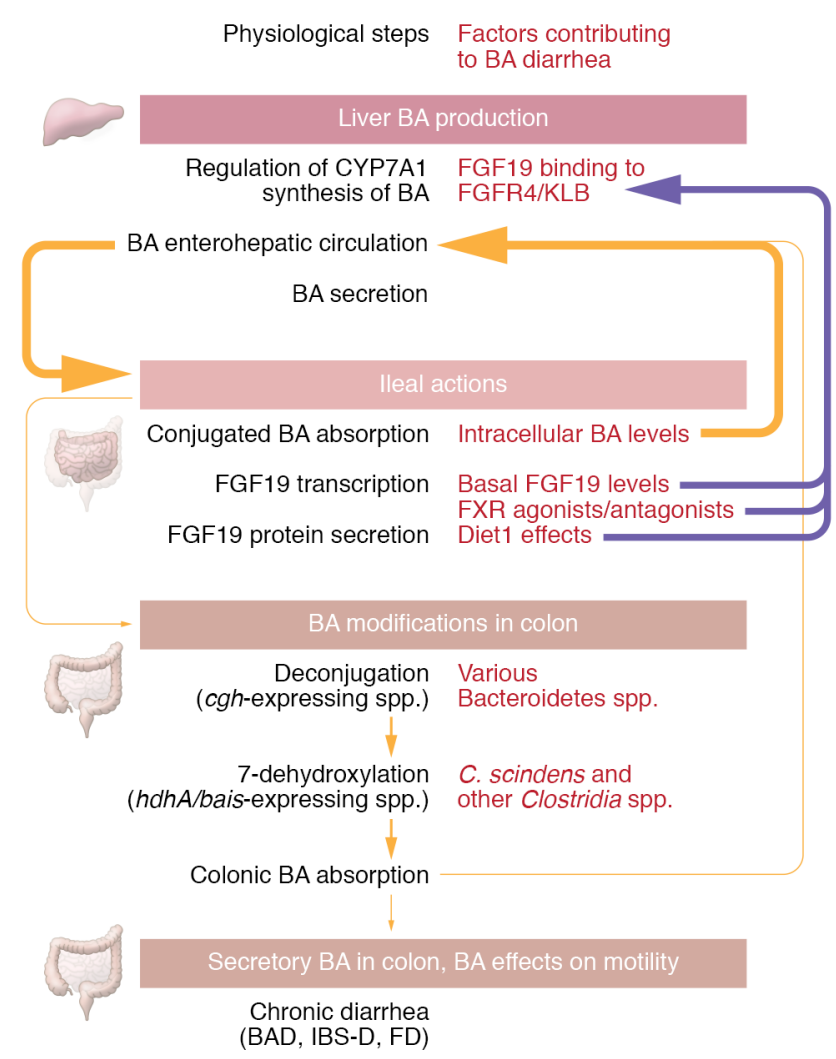

Figure 1. Factors contributing to BA diarrhea. The key physiological steps in BA metabolism produce or interact with specific factors that can contribute to the pathophysiology of altered BA metabolism and thus result in chronic diarrhea. Bacterial species influence various BA proportions (gold arrows with varying width) and influence FGF19 signaling (purple arrow).

by the cgh gene, and 7-dehydroxylation/ dehydrogenation enzymes encoded by bais (7-dehydroxylase) and $h d h A$ (7 $\alpha$-hydroxysteroid dehydrogenase, 7-HSDH) are key to the production of the secondary, modified BAs. Using shotgun metagenomics and DNA extracted from the feces of the study group, Zhao et al. found reduced Bacteroidetes, with decreased abundances of Alistipes and Bacteroides, which express $c g h$, contributing to the reduced activity, and less BA deconjugation in the BA-positive patients (the $\mathrm{BA}^{+} \mathrm{IBS}-\mathrm{D}$ group). This group had an increase in their microbiota in Firmicutes, including Clostridia and especially Clostridium scindens, with significantly higher abundance of 7-dehydroxylation/ dehydrogenation genes. The abundance of C. scindens was positively associated with total fecal BA and serum $\mathrm{C} 4$, and, notably, was inversely related to serum FGF19 levels (16).

A key step for the investigators involved recapitulating these findings in mice. Human fecal microbiota was transplanted into pseudo-germ-free mice, and after one week, those that had received feces from the group of BA-positive patients had cecal microbiota with decreased deconjugating ability and increased 7-HSDH and 7 $\alpha$-dehydroxylating levels. They also had pathophysiologic differences, increased fecal water and $\mathrm{BA}$, increased serum $\mathrm{C} 4$ and hepatic BA-synthesizing enzyme activity, and decreased ileal FGF15 (the mouse homolog of human FGF19). No differences in ileal BA transporters or hepatic receptors were identified. The Firmicutes/Bacteroidetes ratio was higher, and increases in Clostridium cluster XIVa and C. scindens were found (16).

Next, modifications to the microbiota of conventional mice were made. The researchers either directly administered C. scindens into the stomachs or, alternatively, treated the mice with vancomycin, an antibiotic that inhibits Clostridium species. Levels of 7-HSDH and 7a-dehydroxylating activity changed in keeping with the previous findings, as did BA and gene expression. C. scindens enhanced BA synthesis and excretion, reducing ileal
FGF15 at both transcript and protein levels. Conversely, vancomycin increased ileal Fgf15 and inhibited hepatic Cyp7a1 gene expression (16).

Cell-line studies were performed to add to these findings. In the hepatocyte L-02 cell line, vancomycin or $C$. scindens failed to produce direct effects on CYP7A1 expression. In NCI-H716 cells, which have an enterocyte-like phenotype, CDCA stimulated FGF19 expression, as previously demonstrated in similar cells (17), confirming findings with human ileal tissue that FXR agonists potently regulate this gene (13). Further, coincubating the cells with other BAs, including GUDCA, GCDCA, GCA, UDCA, or 7-KDCA, inhibited the CDCA-stimulated FGF19 (16).

Other research has suggested that certain modified, secondary BAs can have actions as FXR antagonists. For example, tauro- $\beta$-muricholic acid was shown to act as an antagonist (18), but humans, unlike mice, have very low levels of this BA. In the Zhao et al. study, the related metabolite, $\omega$-muricholic acid, was detected at higher levels in feces of BA-positive patients. GUDCA also has FXR-antagonist effects, and is increased by metformin, which decreases a specific Bacteroides species and can also cause $\operatorname{BAD}(19,20)$.

\section{Clinical implications}

The findings in Zhao et al. are significant and important, advancing our understanding of the role of the gut microbiota and BAs in IBS-D. They will need to be confirmed in other defined groups of PBAD, such as those diagnosed by reduced SeHCAT retention, blood tests, or by raised fecal total or primary BAs $(6,7)$. They add to previous $\mathrm{PBAD}$ mechanistic studies, where overproduction of BAs with raised $\mathrm{C} 4$, due to impaired FGF19-related negative feedback, are central (6). Reduced deconjugation of BAs gives higher levels of fecal primary BAs (7), but increased 7-dehydroxylation/dehydrogenation produces secondary BAs, which can be reabsorbed and then conjugated. If these conjugated secondary BAs, like GUDCA, inhibit FXR, their increase could explain the reduced ileal FGF19 production.

The microbial and enzymatic changes associated with IBS-D and outlined by Zhao et al. need considering alongside other possi- 
ble factors (Figure 1) (16). For example, lower basal and stimulated FGF19 transcript levels have been shown in patient ileal mucosa explant cultures, implying an inherent defect can predispose individuals to PBAD (14). There is also evidence that the Diet1 protein regulates FGF19 protein secretion, and that DIET1 genetic variants associate with PBAD (21). Further, variants in FGF19 hepatic response genes (FGFR4 and $K L B$ ) may also contribute to PBAD (15).

We now have a target group of organisms, which might be considered to be a biomarker for BAD. This may allow the development of a quick molecular test to detect levels of C. scindens relative to other bacteria in fecal samples. Moreover, since the human population's gut microbiota sits along a continuum with different Firmicutes/Bacteroidetes ratios, it also raises the possibility that we could be able to predict which individuals, enriched with Firmicutes, could have a higher prevalence of PBAD and/or IBS-D with high levels of BA. Is this population of Firmicutes, and specifically the enhanced Clostridia and C. scindens, maintained by the higher BA levels or due to other unknown factors? There is still much work to be done to demonstrate the relevance of these findings and the exact relationships of these microbiota with $\mathrm{BA}$ metabolism and PBAD in individuals with IBS-D. Nevertheless, antibiotic treatment trials, with vancomycin or others targeting Clostridia rather than Bacteroidetes, may be beneficial in PBAD and should be one of the many outcomes of this notable study.

Address correspondence to: Julian Walters, Division of Digestive Diseases, Imperial College London, Hammersmith Hospital,
Du Cane Road, London W12 OHS, United Kingdom. Phone: 44.203.313.2361; Email: julian.walters@imperial.ac.uk.

1. Mearin F, et al. Bowel disorders. Gastroenterology. 2016;150(6):1393-1407.e5.

2. Bajor A, Törnblom H, Rudling M, Ung KA, Simrén $\mathrm{M}$. Increased colonic bile acid exposure: a relevant factor for symptoms and treatment in IBS. Gut. 2015;64(1):84-92.

3. Arasaradnam RP, et al. Guidelines for the investigation of chronic diarrhoea in adults: British Society of Gastroenterology, 3rd edition. Gut. 2018;67(8):1380-1399.

4. Smalley W, Falck-Ytter C, Carrasco-Labra A, Wani S, Lytvyn L, Falck-Ytter Y. AGA clinical practice guidelines on the laboratory evaluation of functional diarrhea and diarrhea-predominant irritable bowel syndrome in adults (IBS-D). Gastroenterology. 2019;157(3):851-854.

5. Bannaga A, Kelman L, O'Connor M, Pitchford C, Walters JR, Arasaradnam RP. How bad is bile acid diarrhoea: an online survey of patientreported symptoms and outcomes. BMJOpen Gastroenterol. 2017;4(1):e000116.

6. Walters JR. Bile acid diarrhoea and FGF19: new views on diagnosis, pathogenesis and therapy. Nat Rev Gastroenterol Hepatol. 2014;11(7):426-434.

7. Vijayvargiya P, Camilleri M. Current practice in the diagnosis of bile acid diarrhea. Gastroenterol ogy. 2019;156(5):1233-1238.

8. Wedlake L, A'Hern R, Russell D, Thomas K, Walters JR, Andreyev HJ. Systematic review: the prevalence of idiopathic bile acid malabsorption as diagnosed by SeHCAT scanning in patients with diarrhoea-predominant irritable bowel syndrome. Aliment Pharmacol Ther. 2009;30(7):707-717.

9. Slattery SA, Niaz O, Aziz Q, Ford AC, Farmer AD. Systematic review with meta-analysis: the prevalence of bile acid malabsorption in the irritable bowel syndrome with diarrhoea. Aliment Pharmacol Ther. 2015;42(1):3-11.

10. Valentin N, et al. Biomarkers for bile acid diarrhoea in functional bowel disorder with diarrhoea: a systematic review and meta-analysis. Gut. 2016;65(12):1951-1959.

11. Hofmann AF, Mangelsdorf DJ, Kliewer SA.
Chronic diarrhea due to excessive bile acid synthesis and not defective ileal transport: a new syndrome of defective fibroblast growth factor 19 release. Clin Gastroenterol Hepatol. 2009;7(11):1151-1154.

12. Walters JR, Tasleem AM, Omer OS, Brydon WG Dew T, le Roux CW. A new mechanism for bile acid diarrhea: defective feedback inhibition of bile acid biosynthesis. Clin Gastroenterol Hepatol. 2009;7(11):1189-1194.

13. Zhang JH, et al. Potent stimulation of fibroblast growth factor 19 expression in the human ileum by bile acids. Am J Physiol Gastrointest Liver Physiol. 2013;304(10):G940-G948.

14. Johnston IM, et al. Characterizing factors associated with differences in FGF19 blood levels and synthesis in patients with primary bile acid diarrhea. Am J Gastroenterol. 2016;111(3):423-432.

15. Camilleri M, et al. Irritable bowel syndromediarrhea: characterization of genotype by exome sequencing, and phenotypes of bile acid synthesis and colonic transit. Am J Physiol Gastrointest Liver Physiol. 2014;306(1):G13-G26.

16. Zhao L, et al. A Clostridia-rich microbiota enhances bile acid excretion in diarrhea-predominant irritable bowel syndrome. J Clin Invest. 2020;130(1):438-450.

17. Zhang Y, LaCerte C, Kansra S, Jackson JP, Brouwer KR, Edwards JE. Comparative potency of obeticholic acid and natural bile acids on FXR in hepatic and intestinal in vitro cell models. Pharmacol Res Perspect. 2017;5(6): e00368.

18. Sayin SI, et al. Gut microbiota regulates bile acid metabolism by reducing the levels of tauro-beta-muricholic acid, a naturally occurring FXR antagonist. Cell Metab. 2013;17(2):225-235.

19. Sun L, et al. Gut microbiota and intestinal FXR mediate the clinical benefits of metformin. Nat Med. 2018;24(12):1919-1929.

20. Appleby RN, et al. Non-alcoholic fatty liver disease is associated with dysregulated bile acid synthesis and diarrhea: A prospective observational study. PLoS One. 2019;14(1):e0211348.

21. Lee JM, et al. Diet1, bile acid diarrhea, and FGF15/19: mouse model and human genetic variants. J Lipid Res. 2018;59(3):429-438. 